\title{
TRICOMAS FOLIARES DE CROTON SECCIÓN BARHAMIA (EUPHORBIACEAE)
}

\author{
Martha Martínez Gordillo ${ }^{1}$ y Silvia Espinosa Matías ${ }^{2}$ \\ ${ }^{1}$ Herbario de la Facultad de Ciencias (FCME), \\ Universidad Nacional Autónoma de México, \\ Apartado postal 70-399, 04510 Coyoacán, México, D.F. \\ ${ }^{2}$ Laboratorio de Microscopía Electrónica de Barrido, Facultad de Ciencias, \\ Universidad Nacional Autónoma de México, 04510 Coyoacán, México, D.F.
}

\section{RESUMEN}

Se hizo un análisis morfológico con microscopía electrónica de barrido de los tricomas presentes en las hojas de 17 especies de la sección Barhamia del género Croton. Se observaron tricomas estrellado-rotados, estrellado-lepidotos, multiradiados y dendríticos, así como glándulas epidérmicas embebidas. Las variaciones morfológicas de los tricomas no permiten caracterizar a la sección de manera inequívoca porque no son exclusivas de la misma, pero se considera que el tipo de tricoma puede ayudar a diferenciar a las especies.

Palabras clave: Barhamia, Croton, Euphorbiaceae, morfología de tricomas.

\begin{abstract}
Trichomes on both leaf surfaces of 17 species of section Barhamia genus Croton were studied using scanning electron microscopy. Stellate-rotate, lepidote-stellate, multiradiate and dendritic trichomes as well as embedded epidermal glands were observed. Based on the morphological variations observed in the section, Barhamia cannot be characterized indisputably by features of the trichomes, although at the species level, trichomes serve as useful distinguishing features.
\end{abstract}

Key words: Barhamia, Croton, Euphorbiaceae, trichome morphology.

\section{INTRODUCCIÓN}

Los tricomas son estructuras importantes dentro de las angiospermas, se presentan con frecuencia y son relativamente fáciles de observar; varían en forma, 
tamaño, distribución y persistencia en las diferentes partes de una planta (Theobald et al., 1979). Se ha postulado que son útiles para proteger a las plantas de elementos ambientales como la radiación solar o el viento, así como de herbívoros que pueden ser dañados físicamente por los tricomas o envenenados por los compuestos químicos que producen (Duke, 1994). El tipo de tricoma puede delimitar especies, géneros y aun familias; sin embargo, en ocasiones, la densidad y los patrones de distribución responden a las condiciones del ambiente o a la edad de la planta, por lo que en algunos casos, su valor taxonómico es discutible (Metcalfe y Chalk, 1950). En este trabajo se examina en detalle la variación morfológica de los tricomas de 17 especies de la sección Barhamia (Klotzsch) Baill. del género Croton con el propósito de obtener caracteres morfológicos útiles para distinguirla.

El género Croton es uno de los más grandes de la familia Euphorbiaceae, Radcliffe-Smith (2001) considera que está conformado por 800 especies, mientras que Webster (1994) señala 1,000 y Govaerts et al. (2000) 1,223. Los miembros de este género pueden ser árboles, arbustos, subarbustos o hierbas y están ampliamente distribuidos en las regiones tropicales del mundo (Webster, 1994). Este género ha sido estudiado desde el punto de vista fitoquímico, en función de la presencia de compuestos carcinogénicos en algunas de sus estructuras (Farnsworth et al., 1969; Moulis et al., 1992; Siems et al., 1992; Amaral y Barnes, 1998; Luna et al., 1999; Fekam et al., 2002); no obstante, los trabajos palinológicos (como los de Punt, 1962 y Nowicke, 1994), los anatómicos (por ejemplo los de Rudall, 1989 y 1994 y Freitas et al., 2001), los citológicos y ecológicos (como los de Domínguez y Bullock, 1989), etc. son relativamente escasos y pocas especies se han estudiado desde otro punto de vista diferente al florístico.

Las especies del género Croton generalmente presentan tricomas estrellados, definidos como estructuras con más de cinco radios que forman un fascículo; cuando los radios están en un solo plano, los tricomas reciben el nombre de rotados, cuando se encuentran sobrepuestos en varios planos, sin que el eje donde se insertan se alargue, se llaman multiangulados, si tienen un radio central erecto son conocidos como porrectos y pueden además ser sésiles o estipitados; si los radios se insertan a diferentes niveles sobre un eje alargado, el tricoma es definido como dendrítico o geminado (Theobald et al., 1979).

Los tricomas lepidotos se presentan con menos frecuencia dentro del género, se caracterizan por presentar un anillo basal conspicuo, donde todos los radios se concentran en un mismo plano y se encuentran unidos en diferentes grados formando una escama. Ocasionalmente se pueden encontrar tricomas simples, que probablemente se originan por supresión del anillo basal de radios (McVaugh, 1961). Entre los tricomas simples y los lepidotos se encuentran formas intermedias 
diferentes, aunque de manera general se han definido como tricomas estrellados y lepidotos, sin mayores precisiones (Webster et al., 1996).

El primer estudio formal de los tricomas en el género Croton fue hecho por Froembling (1896), quien propuso la existencia de cinco tipos básicos: lepidoto, estrellado, fasciculado o rosulado, fasciculado-estipitado y dendrítico. Posteriormente, Webster et al. (1996) intentaron establecer las relaciones filogenéticas entre las secciones propuestas por el mismo Webster (1993) y caracterizarlas por los tricomas presentes en cada una de ellas, reconociendo siete tipos básicos: estrellado, fasciculado, multiradiado y rosulado, dendrítico, lepidoto, papilado y glandular, con varias formas intermedias.

La sección Barhamia (Klotzsch) Baill. es un grupo completamente americano con alrededor de 18 especies, con caracteres muy variables, desde C. betulaster Baill. y C. glutinosus Müll. Arg., con hojas casi glabras y víscidas, que tienen un aspecto brillante, hasta $C$. rhexifolius Baill. con indumento abundante. Las principales características de la sección son: arbustos con hojas generalmente dentadas, sin glándulas conspicuas en el ápice del pecíolo, estípulas glandulares, lobadas o ligeramente disectadas; estambres (5-)8-12, sépalos de las flores pistiladas glandulares marginal y/o abaxialmente y estilos multífidos (Webster,1993). Para esta sección Webster et al. (1996) observaron tricomas estrellado-rotados y porrectos en el haz, con 6-15 radios y tricomas estrellado-rotados con el mismo número de radios en el envés.

\section{MATERIAL Y MÉTODO}

Se analizaron los tricomas del haz y el envés de las hojas de 17 especies de la sección Barhamia (Klotzsch) Baill. del género Croton (ver Apéndice). Los ejemplares identificados como C. muscicapa Mûll. Arg. se consideraron como pertenecientes a dos especies, una de las cuales lleva tal nombre, mientras que la otra se encuentra en proceso de descripción. Se tomaron muestras del haz y el envés de las hojas de material herborizado de ejemplares de las colecciones de los herbarios CICY, CIQRO, D, F, MEXU, LL, NY, P, R, RB, SPF, UB, US y XAL.

Las muestras se montaron sobre cinta conductiva de carbón de doble cara en portamuestras de aluminio. Posteriormente se cubrieron con una fina capa de oro en una ionizadora Denton Vacum Desk II. Las observaciones y toma de fotografías se realizaron en un microscopio electrónico de barrido Jeol JSM$5310 \mathrm{LV}$. 


\section{RESULTADOS}

En las hojas de las especies de Croton sección Barhamia (Klotzsch) Baill. (Cuadros 1 y 2), se encuentran tricomas rotados (Fig. 1), multiradiados (Fig. 2) y ocasionalmente dendríticos, como en el caso del envés de Croton urticifolius Lam. (Fig. 3). También se observan tricomas estrellado-lepidotos, con los radios unidos desde el centro en 15-30\% de su longitud, en el envés de C. ameliae Lundell (Fig. 4). El número de radios varía de 5 a 21 (Cuadros 1 y 2), siendo los tricomas multiradiados los que presentan un mayor número de los mismos. Una característica de todos los tricomas de las especies observadas es la presencia de un radio central erecto, es decir son porrectos; este radio puede ser más corto (Fig. 5), más largo (Fig. 6) o de longitud similar al resto de los radios (Fig. 7); en ocasiones esta característica puede presentarse mezclada, como sucede en $C$. agoensis Baill. y $C$. urticifolius Lam., en donde se observan tricomas con radio central corto y tricomas con el radio central largo (Fig. 8). Croton essequiboensis Klotzsch y C. adenocalix Baill. se caracterizan por la presencia de una célula basal, conspicua y aplanada (Fig. 9).

Todas las especies examinadas presentan glándulas epidérmicas embebidas sobre la lámina foliar, tanto en el haz como en el envés (Fig. 10). En Croton glutinosus Müll. Arg., C. betulaster Baill., C. muscicapa Müll. Arg. y C. sp. nov. la secreción de estas glándulas cubre totalmente la lámina foliar (Fig. 11). También se observan tricomas glandulares en el margen de la hoja y sobre la cresta de los dientes como en C. muscicapa Müll. Arg. (Fig. 12).

\section{DISCUSIÓN Y CONCLUSIONES}

Webster et al. (1996) definen los tipos de tricomas de cada sección y registran tricomas estrellado-rotados, porrectos en el haz y estrellado-rotados, no porrectos en el envés como característicos de la sección Barhamia (Klotzsch) Baill. Además de los tipos señalados por estos autores, en el presente trabajo se observan dos formas de tricoma adicionales dentro de la sección: tricomas multiradiados en el haz y envés de Croton urticifolius Lam., que en algunos ejemplares son dendríticos, similares a los de las especies de las secciones Velamea Baill. y Eremocarpus (Benth.) G. L. Webster, y tricomas estrellado-lepidotos con los radios unidos desde el centro entre 15 y $30 \%$ de su longitud, en el envés de $C$. ameliae Lundell, los cuales son similares a los encontrados en especies de las secciones Luntia (Raf.) G. L. Webster y Andrichnia Baill.

Los tricomas de las hojas del género Croton son variados en cuanto a tamaño, número de radios y unión de los mismos. El hecho de presentar tricomas con el 
Martinez y Espinosa: Tricomas foliares de Croton Sección Barhamia

\begin{tabular}{|c|c|c|c|c|c|c|c|c|c|c|c|c|c|c|c|c|c|}
\hline 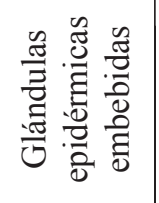 & 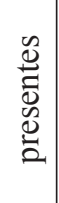 & 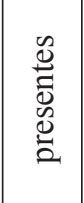 & 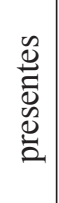 & $\begin{array}{l}0 \\
0 \\
0 \\
0 \\
0 \\
0 \\
0\end{array}$ & $\begin{array}{l}0 \\
0 \\
0 \\
0 \\
0 \\
0 \\
0\end{array}$ & $\mid \begin{array}{l}0 \\
0 \\
0 \\
0 \\
0 \\
0 \\
0 \\
0\end{array}$ & $\mid \begin{array}{l}0 \\
0 \\
0 \\
0 \\
0 \\
0 \\
0 \\
0\end{array}$ & $\begin{array}{l}0 \\
0 \\
0 \\
0 \\
0 \\
0 \\
0 \\
0\end{array}$ & 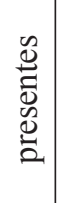 & 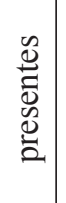 & 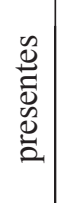 & 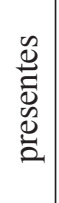 & 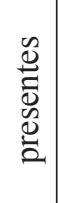 & $\mid \begin{array}{l}0 \\
0 \\
0 \\
0 \\
0 \\
0 \\
0 \\
0\end{array}$ & $\mid \begin{array}{l}0 \\
0 \\
0 \\
0 \\
0 \\
0 \\
0 \\
0\end{array}$ & 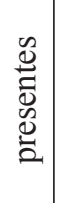 & 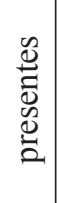 \\
\hline 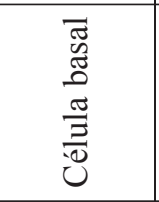 & 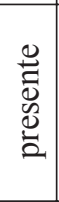 & 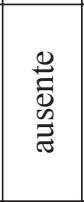 & 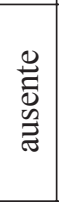 & 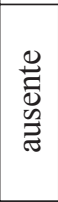 & 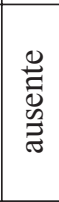 & 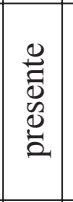 & 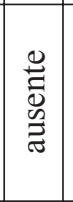 & 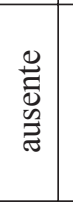 & 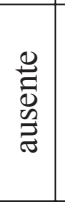 & 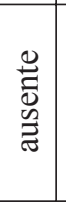 & $\begin{array}{l}\stackrel{0}{0} \\
\overline{0} \\
\text { 岕 }\end{array}$ & 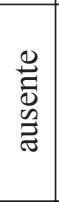 & 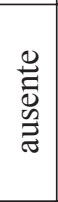 & 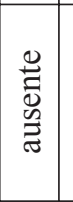 & 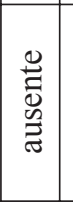 & 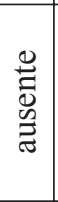 & 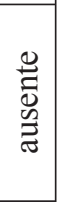 \\
\hline 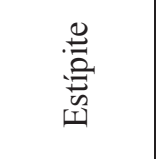 & 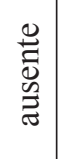 & 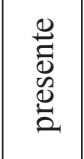 & 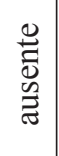 & 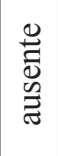 & 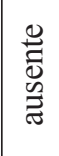 & 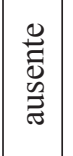 & 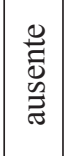 & 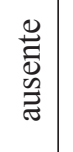 & 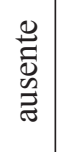 & 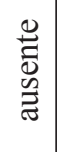 & 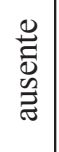 & 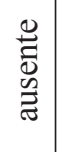 & 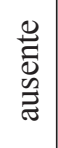 & 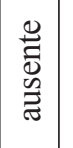 & 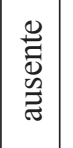 & 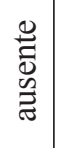 & 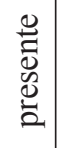 \\
\hline 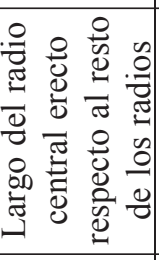 & $\begin{array}{l}0 \\
\frac{0}{0} \\
. \overline{0} \\
> \\
>\end{array}$ & 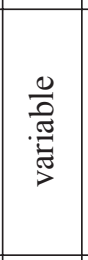 & 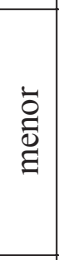 & & 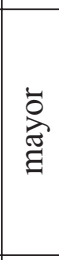 & $\begin{array}{l}\dot{\overline{0}} \\
\overline{0} \\
\dot{\Xi}\end{array}$ & 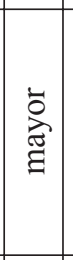 & 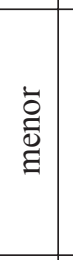 & 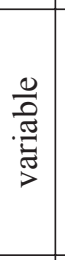 & & $\overrightarrow{\widetilde{J}}$ & & 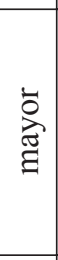 & 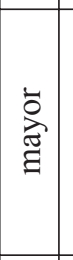 & 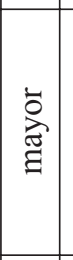 & 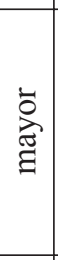 & 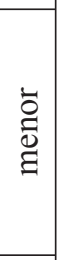 \\
\hline 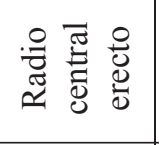 & 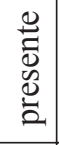 & 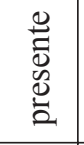 & $\begin{array}{l}0 \\
\stackrel{0}{0} \\
0 \\
0 \\
0\end{array}$ & 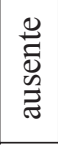 & 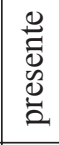 & 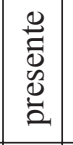 & $\begin{array}{l}0 \\
\stackrel{0}{0} \\
0 \\
0 \\
0 \\
\end{array}$ & 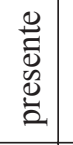 & $\begin{array}{l}0 \\
\stackrel{0}{0} \\
0 \\
0 \\
\vdots\end{array}$ & 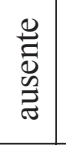 & 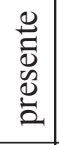 & $\begin{array}{l}0 \\
\stackrel{0}{0} \\
0 \\
\text { ثे }\end{array}$ & 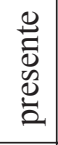 & \begin{tabular}{|l|}
0 \\
0 \\
0 \\
0 \\
0 \\
0 \\
0 \\
\end{tabular} & \begin{tabular}{|l|}
0 \\
0 \\
0 \\
0 \\
0 \\
0 \\
0
\end{tabular} & 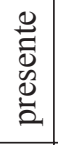 & 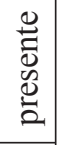 \\
\hline 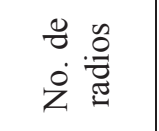 & $\stackrel{0}{\sim}$ & $\stackrel{\infty}{0}$ & $\infty$ & $\begin{array}{l}0 \\
i\end{array}$ & $\tilde{\sigma}^{\circ}$ & $\begin{array}{l}\infty \\
- \\
-\end{array}$ & $\mid \begin{array}{l}0 \\
\infty\end{array}$ & $\hat{0}$ & 0 & 0 & $r$ & $r$ & $\infty$ & $\infty$ & $\infty$ & $\infty$ & $\infty$ \\
\hline$\stackrel{\varrho}{气}$ & 胥 & 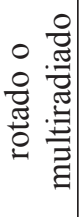 & $\begin{array}{l}0 \\
\stackrel{\pi}{0} \\
0\end{array}$ & $\begin{array}{l}\stackrel{0}{0} \\
\stackrel{\pi}{0} \\
=\end{array}$ & 茜 & $\begin{array}{l}\stackrel{8}{0} \\
\substack{0 \\
0}\end{array}$ & 量 & & 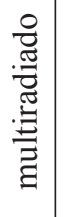 & 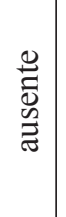 & $\begin{array}{l}\frac{0}{0} \\
\frac{\pi}{0} \\
0\end{array}$ & $\begin{array}{l}\frac{0}{\pi} \\
\frac{\pi}{0} \\
\end{array}$ & $\begin{array}{l}\frac{0}{\pi} \\
\stackrel{\pi}{0} \\
0\end{array}$ & $\mid \begin{array}{l}0 \\
\frac{0}{\pi} \\
\stackrel{\pi}{0} \\
: 1\end{array}$ & 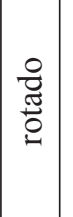 & $\begin{array}{l}\frac{0}{\pi} \\
\stackrel{\pi}{0} \\
0\end{array}$ & 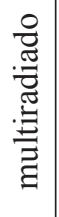 \\
\hline $\begin{array}{l}\frac{0}{3} \\
\frac{0}{0} \\
0 \\
0.1\end{array}$ & 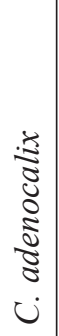 & 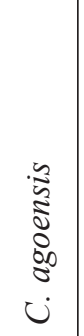 & 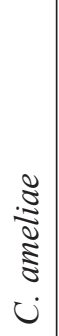 & 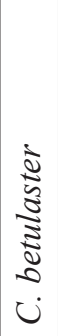 & 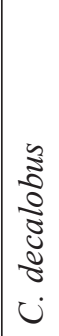 & 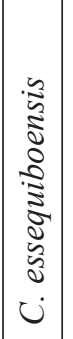 & $\mid \begin{array}{c}0 \\
\vdots \\
\vdots \\
0 \\
0 \\
0 \\
0 \\
0 \\
0 \\
0 \\
0\end{array}$ & 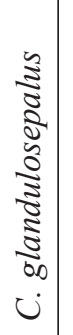 & 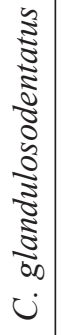 & $\begin{array}{c}5 \\
\vdots \\
\vdots \\
\vdots \\
\vdots \\
0 \\
0 \\
ن\end{array}$ & 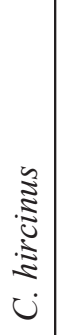 & 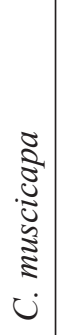 & $\begin{array}{l}\dot{a} \\
\dot{a} \\
\dot{2} \\
\dot{0}\end{array}$ & 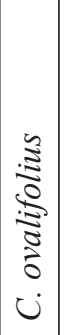 & 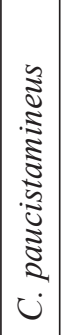 & 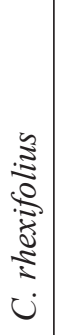 & 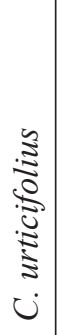 \\
\hline
\end{tabular}




\begin{tabular}{|c|c|c|c|c|c|c|c|c|c|c|c|c|c|c|c|c|c|}
\hline 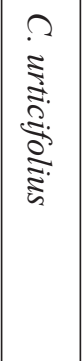 & 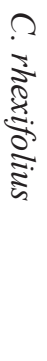 & 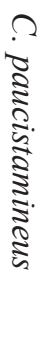 & חל & $\begin{array}{l}\Omega \\
0 \\
0 \\
0 \\
0\end{array}$ & 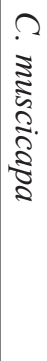 & 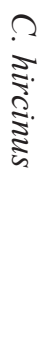 & 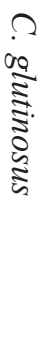 & 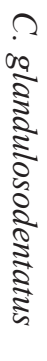 & 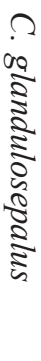 & 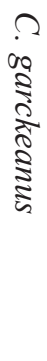 & 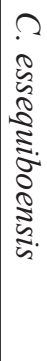 & 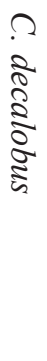 & 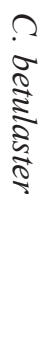 & 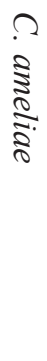 & 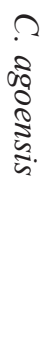 & 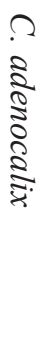 & $\begin{array}{l}\frac{1}{n} \\
\frac{n}{0} \\
\stackrel{0}{0} .\end{array}$ \\
\hline 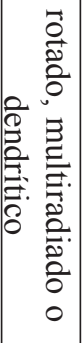 & $\begin{array}{l}\overrightarrow{0} \\
\text { مै } \\
\text { Oे }\end{array}$ & 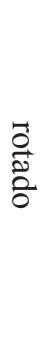 & 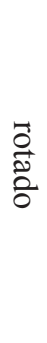 & 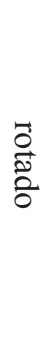 & 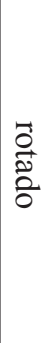 & $\begin{array}{l}\overrightarrow{0} \\
\text { مै } \\
\text { Oे }\end{array}$ & 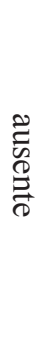 & 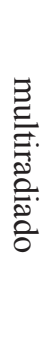 & 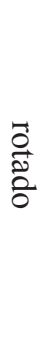 & 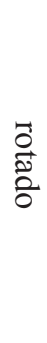 & 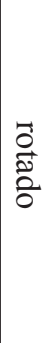 & $\begin{array}{l}\overrightarrow{0} \\
\text { 泀 } \\
\text { Oे }\end{array}$ & $\begin{array}{l}\overrightarrow{0} \\
\text { 苛 } \\
\text { On }\end{array}$ & 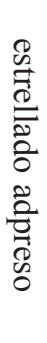 & 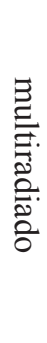 & $\begin{array}{l}\overrightarrow{0} \\
\text { مै } \\
\text { : }\end{array}$ & 쿵 \\
\hline 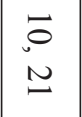 & 6 & $\infty$ & $\checkmark$ & $\stackrel{r}{\infty}$ & $a$ & 6 & 0 & 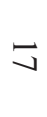 & 二 & $\begin{array}{l}0 \\
0 \\
0\end{array}$ & $\frac{\infty}{0}$ & $\checkmark$ & u & $\frac{\infty}{0}$ & $\frac{a}{i}$ & $\infty$ & 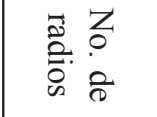 \\
\hline 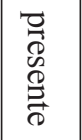 & 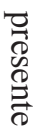 & 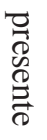 & 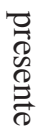 & $\begin{array}{l}\text { 응 } \\
0 \\
0 \\
\stackrel{0}{0} \\
\stackrel{0}{0}\end{array}$ & $\begin{array}{l}\text { 듕 } \\
\stackrel{8}{\infty} \\
\stackrel{0}{0} \\
\stackrel{0}{0}\end{array}$ & 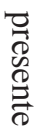 & 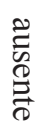 & 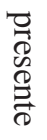 & 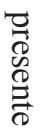 & $\begin{array}{l}\text { 듕 } \\
\stackrel{8}{\infty} \\
\stackrel{0}{0} \\
\stackrel{0}{0}\end{array}$ & 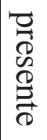 & $\begin{array}{l}\text { D } \\
\mathbb{D} \\
\stackrel{0}{0} \\
\stackrel{0}{0}\end{array}$ & $\begin{array}{l}\stackrel{0}{0} \\
\stackrel{0}{:} \\
\stackrel{0}{0}\end{array}$ & 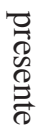 & $\begin{array}{l}\text { 뭉 } \\
0 \\
0 \\
\stackrel{0}{0} \\
\stackrel{0}{0}\end{array}$ & 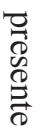 & 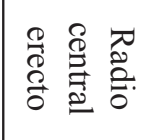 \\
\hline 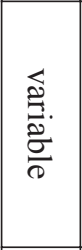 & 岛. & $\stackrel{\overrightarrow{09}}{\stackrel{0}{2}}$ & 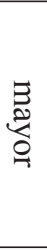 & 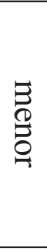 & 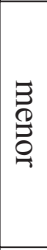 & 胥 & & 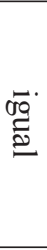 & $\begin{array}{l}3 \\
0 \\
0 \\
0\end{array}$ & 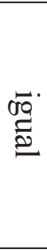 & $\stackrel{\overrightarrow{0 Q}}{\stackrel{0}{0}}$ & $\begin{array}{l}\bar{\Xi} \\
\stackrel{9}{0} \\
0\end{array}$ & & $\begin{array}{l}3 \\
\overparen{0} \\
\stackrel{0}{0}\end{array}$ & 苛. & 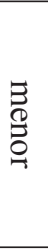 & 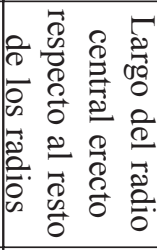 \\
\hline 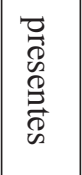 & 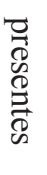 & 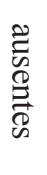 & 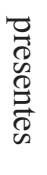 & 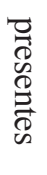 & 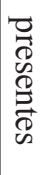 & 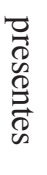 & 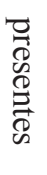 & 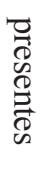 & $\begin{array}{l}\stackrel{2}{0} \\
\stackrel{0}{0} \\
\stackrel{0}{\vec{P}}\end{array}$ & 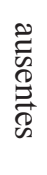 & 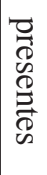 & 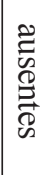 & 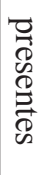 & 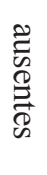 & 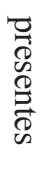 & 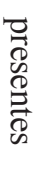 & 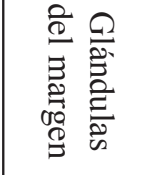 \\
\hline 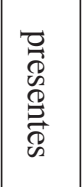 & 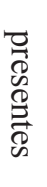 & 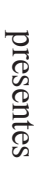 & 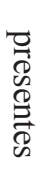 & 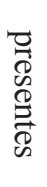 & 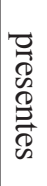 & 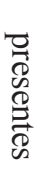 & 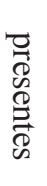 & 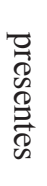 & 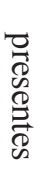 & & 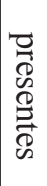 & 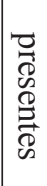 & 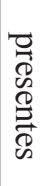 & 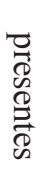 & 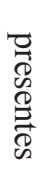 & 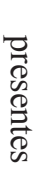 & 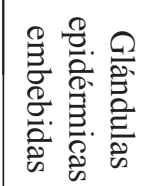 \\
\hline
\end{tabular}



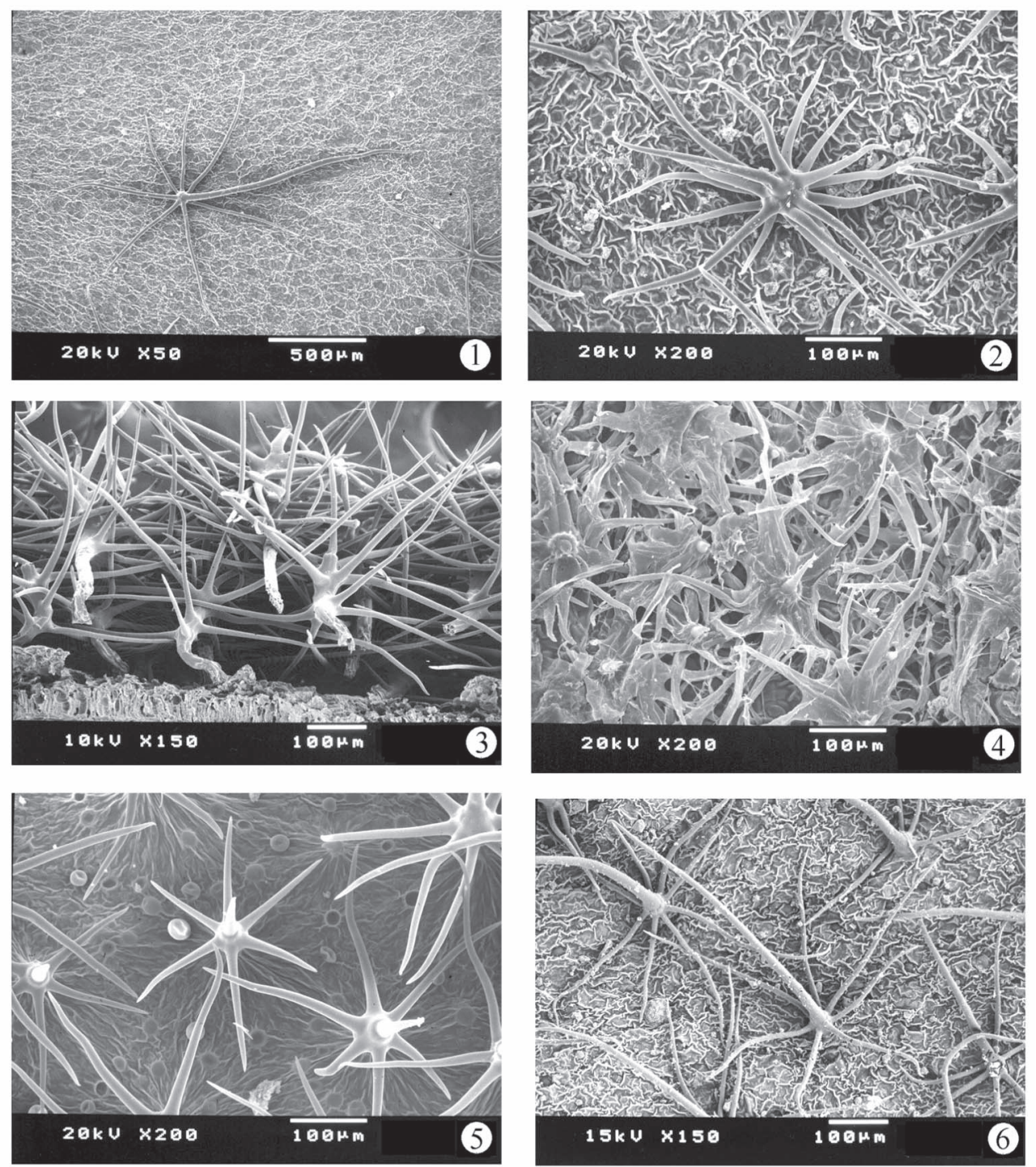

Figs. 1-6. 1. Tricoma estrellado-rotado sobre el haz de Croton ovalifolius. 2. Tricoma multiradiado sobre el envés de $C$. glandulosodentatus. 3 . Tricomas dendríticos y estipitados en el envés de $C$. urticifolius. 4. Tricomas estrellado-lepidotos sobre el envés de C. ameliae. 5. Tricomas estrellado-rotados en el envés de $C$. decalobus con radios erectos pequeños. 6 . Tricomas estrellado-rotados del haz de $C$. rhexifolius con el radio erecto más largo. 

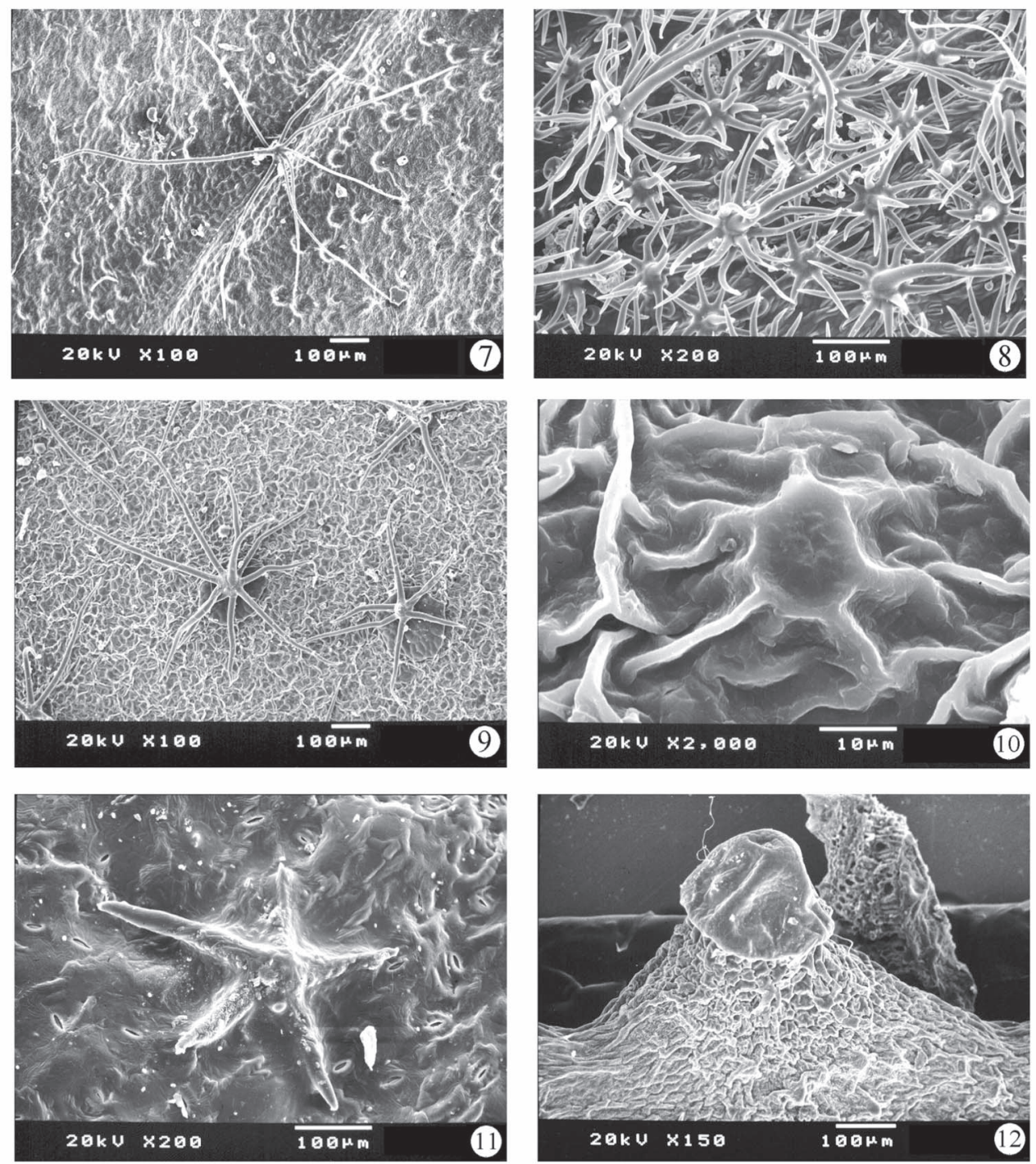

Figs. 7-12. 7. Tricoma estrellado-rotado en el haz de Croton muscicapa con el radio erecto similar al resto de los radios. 8. Tricomas multiradiados en el haz de C. agoensis con radios erectos, pequeños y largos mezclados. 9. Tricomas estrellado-rotados en el haz de $C$. adenocalix con una célula aplanada y grande en la base. 10. Glándula epidérmica embebida en el haz de C. glandulosepalus. 11. Superficie adaxial de la hoja de C. betulaster con secreción de glándulas cubriendo totalmente la lámina. 12. Glándula marginal sobre el diente en $C$. muscicapa. 
radio central erecto con diferente longitud, en ambas superficies de la hoja, como en C. adenocalix Baill. C. glandulosodentatus Pax et K. Hoffm. C. urticifolius Lam. y $C$. agoensis Baill., parece estar relacionado con el grado de madurez del tricoma.

Webster (1933) incluye a Croton agoensis Baill. dentro de la sección Barhamia, sin embargo la presencia de caracteres tales como glándulas infundibuliformes en la unión de la lámina y el pecíolo (semejante a las especies de las secciones Cyclostigma Griseb., Cleodora (Klotzsch) Baill. y Ocalia (Klotzsch) Baill.), así como de glándulas estipitadas del margen de la hoja (como en las especies de la sección Adenophyllum), asociadas al seno de los dientes (como en las especies de la sección Ocalia (Klotzsch) Baill.), hacen dudosa su pertenencia a la sección Barhamia (Klotzsch) Baill., ya que las especies de esta última no presentan los caracteres descritos. Su inclusión o no dentro de la misma aún está por determinarse y para ello se está llevando a cabo en la actualidad un análisis filogenético.

En Croton, las glándulas epidérmicas sobre la lámina foliar se han señalado como carácter distintivo de la sección Lasiogyne (Klotzsch) Baill.; en el presente trabajo se observa que estas glándulas son comunes sobre el haz y el envés y que, junto con las del margen de la hoja están relacionadas con la producción y secreción de metabolitos secundarios, principalmente terpenos (Fansworth et al., 1969), que le confieren el olor característico y que evita la herbivoría en muchas de las especies (Martínez-Gordillo, obs. pers.). En Croton glutinosus Müll. Arg., C. muscicapa Müll. Arg., C. sp. nov. y en C. betulaster Müll. Arg. las láminas foliares están cubiertas con gran cantidad de este tipo de secreciones, lo que dificulta la observación de las estructuras sobre las mismas. Tales glándulas se encuentran también en las secciones Micranthis Baill. y Medea (Klotzsch) Baill. (MartínezGordillo, obs. pers.), lo que no permite usarlas como un carácter para diferenciar secciones cercanas.

La persistencia y la densidad de los tricomas son parámetros de valor discutible, porque dependen de las condiciones del ambiente y de la edad de la planta (Metcalfe y Chalk, 1950). En Croton la persistencia de los tricomas no es constante, ya que en muchas especies las hojas son pelosas cuando jóvenes y carecen de tricomas en la madurez, presentando la condición llamada glabrescente, mientras que la densidad varía dependiendo de la época de colecta y de la edad de las plantas. Este fenómeno es particularmente manifiesto en $C$. adspersus Benth., de la sección Velamea Baill., que en la forma de verano presenta hojas glabrescentes y en la de invierno hojas tomentosas (Martínez-Gordillo, obs. pers.). Las variaciones en la densidad de los tricomas han conducido a sinonimias, por lo que este carácter no es considerado útil para delimitar especies. Lo anterior también se observa en ejemplares de C. muscicapa Müll. Arg., pues algunos presentan el envés tomentoso mientras que otros tienen ambas superficies de la lámina casi glabras. 
En conclusión, las características que se observan sugieren que, aunque los rasgos morfológicos de los tricomas no pueden usarse como caracteres únicos para definir a la sección, la combinación de tipos de tricomas en el haz y envés son útiles para distinguir a las diferentes especies. Además se encontraron algunos caracteres previamente desconocidos para la sección, como la célula basal en los tricomas de Croton essequiboensis Klotzsch y C. adenocalix Baill., los tricomas dendríticos y estipitados, similares a los de C. suberosus Kunth (sección Velamea Baill.), en el envés de C. urticifolius Lam., las glándulas embebidas, ampliamente distribuidas en la sección, y los tricomas estrellado adpresos del envés de $C$. ameliae Lundell. Por otro lado es importante resaltar que las investigaciones anatómicas en el género y la familia Euphorbiaceae son escasas, aun cuando son una fuente de caracteres necesarios para entender a Croton integralmente.

\section{AGRADECIMIENTOS}

Agradecemos al Biól. José Antonio Hernández y al M. en C. Alejandro Martínez Mena, del laboratorio de Microcine, de la Facultad de Ciencias de la Unversidad Nacional Autónoma de México, por la captura en imagen digital de las fotografías del microscopio electrónico de barrido y la asesoría y apoyo técnico y a la Dra. Sonia Vázquez Santana por la revisión del manuscrito.

\section{LITERATURA CITADA}

Amaral, A. C. F. y R. A. Barnes. 1998. A tetrahydroprotoberberine alkaloid from Croton hemiargyreus. Phytochemistry 47: 1445-1447.

Domínguez, C. A. y S. H. Bullock. 1989. La reproducción de Croton suberosus (Euphorbiaceae) en luz y sombra. Revista Biol. Trop. 37: 1-10.

Duke, S. O. 1994. Glandular trichomes -A focal point and structural interactions. Int. J. Plant. Sci. $155: 617-620$.

Farnsworth, N. R., R. N. Blomstery y W. M. Messmer. 1969. A phytochemical and biological review of the genus Croton. Lloydia 32:-1-28.

Fekam, F., F. Keumedjio, P. M. Jazet, B. T. Ngadjui, P. H. Amvam, C. Menut y J. M. Bessiere. 2002. Essential oils from Croton zambesicus Müell. Arg. growing in Cameroon. Flavour and Fragance J. 17: 215-217.

Freitas, L., G. Bernardello, J. Galetto y A. A. S. Paoli. 2001. Nectaries and reproductive biology of Croton sarcopetalus (Euphorbiaceae). Bot. J. Linn. Soc. 136: 267-277.

Froembling, W. 1896. Anatomisch-systematische Untersuchung von Blatt un Axe der Crotoneen und Euphyllantheen. Bot. Zentralbl. 65: 129-442.

Govaerts, R. D., D. Frodin y A. Radcliffe-Smith. 2000. World checklist and bibliography of Euphorbiaceae. The Royal Botanic Gardens, Kew. Inglaterra. pp. 417-536. 
Luna, A. M., J. C. R. Silva, A. R. Campos, V. S. N. Rao, M. A. M. Maciel y A. C. Pinto. 1999. Antioestrogenic effect of trans-dehydrocrotonin, a nor-clerodane diterpene from Croton cajucara Benth. in rats. Phytotherapy Res. 13: 689-691.

McVaugh, R. 1961. Euphorbiaceae novae novo-galicianae. Brittonia 13: 145-205.

Metcalfe, C. R. y L. Chalk. 1950. Anatomy of the dicotyledons. Vol. II. Clarendon Press. Oxford. pp. 1207-1235.

Moulis, C., M. Bon, J. Jaud y I. Fouraste. 1992. Crovatin, a furanoid diterpene from Croton levatii. Phytochemistry 31: 1421-1423.

Nowicke, J. W. 1994. A palynological study of Crotonoideae (Euphorbiaceae). Ann. Missouri Bot. Gard. 81: 245-269.

Punt, W. 1962. Pollen morphology of Euphorbiaceae with special reference to taxonomy. Wentia 7: 1-116.

Radcliffe-Smith, A. 2001. Genera Euphorbiacearum. The Royal Botanic Gardens, Kew. Inglaterra. $455 \mathrm{pp}$.

Rudall, P. 1989. Laticifers in vascular cambium and wood of Croton spp. (Euphorbiaceae). I.A.W.A. Bull. 10: 379-383.

Rudall, P. 1994. Laticifers in Crotonoideae (Euphorbiaceae): Homology and evolution. Ann. Missouri Bot. Gard. 81: 270-282.

Siems, K., X. A. Domínguez y J. Jakupovic. 1992. Diterpenes and other constituents from Croton cortesianus. Phytochemistry 31: 2055-2058.

Theobald, W. L., J. L. Krahulik y R. C. Rollings, 1979. Trichome description and classification. In: Metcalfe, C. R. y L. Chalk (eds.). Anatomy of the dicotyledons. Vol. I. Clarendon Press. Oxford. pp. 40-53.

Webster, G. L. 1993. A provisional synopsis of the sections of the genus Croton (Euphorbiaceae). Taxon 42: 793-823.

Webster, G. L. 1994. Synopsis of the genera and suprageneric taxa of Euphorbiaceae. Ann. Missouri Bot. Gard. 81: 33-144.

Webster, G. L., M. J. del Arco-Aguilar y B. A. Smith. 1996. Systematic distribution of foliar trichome types in Croton (Euphorbiaceae). Bot. J. Linn. Soc. 121: 41-57. 
Apéndice. Lista de especímenes utilizados para las observaciones de los tricomas foliares.

Croton adenocalix Baill.

Brasil, Ceará: Parque Nacional de 7 cidades, Fernandez 8146 (D).

C. agoensis Baill.

Brasil, Bahia: Espigao Mestre, Anderson 36453, 37068 (RB); Goias, Serra Dos Cristais, Cristalina, Irwin 31348 (RB).

C. ameliae Lundell

México, Yucatán: a $6 \mathrm{~km}$ al O de Timum, sobre el camino Dzitas, Cabrera 11257

(CICY); Chichén Itzá, Lundell y Lundell 7447 (LL).

C. betulaster Müll. Arg.

Brasil, Palmeiras: Pai Inácio, Mori 13214 (D).

C. decalobus Müll. Arg.

México, Veracruz: mpio. Cuitláhuac, 3 km al NO de Cuitláhuac en caminos de terracería, Hansen y Nee 7564 (XAL).

C. essequiboensis Klotzsch

Brasil, Roraima: Boa Vista para Rom Fim, Rio Arraia, peto de Guiana. Ponto 8. Pires y Leite 14690 (D); Venezuela, Bolívar: Hato El Diamante, aprox. $15 \mathrm{~km}$ al SE de El Manteco, Huber y Alarcón 6509 (D).

C. garckeanus Müll. Arg.

Brasil, Santa Catarina: mpio. Porto União, east of Valões (Irineópolis) on the road to Canoinhas, Smith y Reitz 8635 (R); Valões, Reitz y Klein 13548 (US).

C. glandulosepalus Millsp.

México, Quintana Roo: mpio. Carrillo Puerto, Reserva Sian'Ka'an, km. 23 de Vigía a Carrillo, Durán y Olmsted 980 (CIQRO); Yucatán: Chichén Itzá, Steere 1487 (F).

C. glandulosodentantus Pax et K. Hoffm.

Brasil, Ceará: Guaraciaba, do Norte Ceará, Fernández 7897 (D); Tapera Pernambuco, Pickel 1469 (US).

C. glutinosus Müll. Arg.

Brasil, Bahia: mpio. Piatã, estrada entre Piatã y Abaira, a 3 km ao S de Piatã, Mori 13391 (NY); Rio de Contas, $1 \mathrm{~km}$ da cidade na estrada para Marcolino Moura, Pirani 2151 (SPF). 
Apéndice. Continuación.

C. hircinus Vent.

Panamá, Coclé: road to El Copé from interamerican higway, Burch 1370 (D); between El Copé y Barrigón, Sytsma 1750 (F).

C. muscicapa Müll. Arg.

Brasil, Bahia: mpio. Morro do Chapeau, entres Fazendas Efigenia e Boa Vista de Sao José, Sarmento y Bautista 863 (RB); Minas Gerais: Planalto, 15 km of Grão Mogol, Irwin 235218 (UB).

C. ovalifolius Vahl

Costa Rica, Guanacaste: $29 \mathrm{mi} \mathrm{N}$ de Liberia, Webster et al. 12475 (MEXU).

C. paucistamineus Müll. Arg.

Brasil, Mato Grosso: mpio. Miranda, $30 \mathrm{~km}$ al O de Miranda, Allem 698 (D)

C. rhexifolius Baill.

Brasil, Minas Geraes, Saint-Hilaire 1560 (P).

C. sp. nov.

Brasil, Bahia: mpio Andorai, Sierra de Andorai, Capa Bode, entrada por Mucugé, campos rupestres, Martinelli, 5417 (RB); mpio. Lençois, a lo largo BR 242, a $15 \mathrm{~km}$ al NO de Lençois, km 225, campo rupestre, Mori y Boom 14258 (NY).

C. urticifolius Lam.

Brasil, Rio de Janeiro: mpio. Maricá, Itaipuacu, serra do Tiririca, alto do morro no limite com municipio de Niteroi, Pirani y Kallunki 3563 (NY); Boia, ca 1 km S of Rio Contas, on side road to $\mathrm{W}$ of the road to libramiento do Brumado, Harley 15070 (NY). 